\title{
Remote Fitting Procedures for Upper Limb 3D Printed Prostheses
}

Jorge M. Zuniga ${ }^{1,2}$, Keaton J. Young ${ }^{1}$, Jean L. Peck ${ }^{3}$, Rakesh Srivastava ${ }^{4}$, James E. Pierce ${ }^{1}$, Drew R. Dudley ${ }^{1}$, David A. Salazar ${ }^{1}$, and Jeroen Bergmann ${ }^{5}$.

1. University of Nebraska at Omaha, Department of Biomechanics, USA

2. Facultad de Ciencias de la Salud, Universidad Autónoma de Chile, Chile

3. CHI Health Creighton University Medical Center and an adjunct faculty at the Department of Occupational Therapy at Creighton University, USA

4. Innovative Prosthetics \& Orthotics, USA

5. Department of Engineering Science, University of Oxford, UK.

Corresponding Author:

Jorge M. Zuniga Ph.D.

Department of Biomechanics

Biomechanics Research Building

3D Printed Prosthetic, Orthotic \& Assistive Devices

University of Nebraska at Omaha

Email: jmzuniga@unomaha.edu 


\section{Abstract}

2 11 study.

12 Results: The research participants reported a score of $3.92 \pm 0.50$ (closer to the statement "quite

Objective: The objective of the current investigation was twofold: i) described a remote fitting procedure for upper limb 3D printed prostheses and ii) assess patient satisfaction and comfort with 3D printed prostheses fitted remotely.

Design: A qualitative study using content and score analysis to describe patient satisfaction after 4 weeks of using an upper limb 3D printed prosthesis fitted remotely. The novel remote fitting procedure is described in detail.

Subjects: Six children (three girls and three boys, 6 to 16 years of age) and 2 adults (males of 25 and 59 years of age) with congenital $(n=7)$ and acquired $(n=1)$ upper limb loss participated in this

Resuls: The research paticipats reported a score of $3.92 \pm 0.50$ (closer to the stanent "quite satisfied") for the device satisfaction section of the QUEST questionnaire (Table 2). This acceptable level of satisfaction of our research participant reported in the QUEST was confirmed by the agreement scores of the OPUS items related to prosthetic fitting $($ My prosthesis fits well $=$ $4.13 \pm 0.50$ ) and comfort (My prosthesis is comfortable throughout the day $=3.57 \pm 0.98$ ). Furthermore, the comfort level rating in the general prosthetic survey resulted in a score of $3.75 \pm$ 0.70 (closer to the statement "the prosthetic device feels comfortable") confirming the results of the QUEST and OPUS.

Conclusions: The ability to fit an upper-limb prosthesis remotely, represents a promising methodology to fit upper-limb 3D printed prostheses for patients from developing countries or rural areas. The increasing availability of smartphones and other digital devices makes it possible to obtain photographs from patients located in rural areas that have little or no access to trained technicians. These photographs along with the cost-effective desktop 3D printers allows for the 
1 extraction of the anthropometric measurements required for the development of a 3D printed upper

2 limb prosthesis remotely.

3

4 Keywords: Computer-aided design-computer-aided manufacturing, rapid prototyping, upper-

5 limb prosthetics, prosthetic design, patient satisfaction 
1

2 The Centers for Disease Control and Prevention (CDC) estimates that about 1,500 babies are born

3 with upper-limb reductions every year in the U.S. ${ }^{1,2}$ In other parts of the world, such as Australia,

4 Finland, and Canada reports indicate that 3.4 to 5.3 of 10,000 live-born children suffer upper-limb

5 anomalies. ${ }^{3}$ However, in the United States there are many more unreported cases due to the lack

6 of a mandatory reporting system of birth defects and child amputees. Children with congenital

7 unilateral reductions are most frequently seen in pediatric amputation clinics. Only 1 in 9,400

8 children and adults are considered for prosthetic fitting due to the complexity of the reduction and

9 fitting procedures, as well as the lack of interest of the child in using a prosthesis. ${ }^{3-5}$ Even with the

10 great advances in prosthetic technology, a large number of individuals with upper limb reductions

11 still express dissatisfaction with the available technology. ${ }^{4,6} 7$ Factors that contribute to this

12 discontent include late age of fitting, high complexity of the devices and fitting procedures, as well

13 as high costs of devices and repairs. ${ }^{4,8,9}$ In addition, $35 \%$ to $58 \%$ of children with access to

14 prostheses reject them due to excessive weight, lack of functional use, discomfort, and low visual

15 appeal of the device. ${ }^{4,} 8-10$ Recent technological advances in computer-aided design (CAD)

17 designing, and manufacturing low-cost and customized upper-limb 3D printed prostheses

18 remotely. ${ }^{11,12}$ However, there is currently only one paper describing the remote fitting procedures

19 for upper limb 3D printed prosthesis. Zuniga et al., ${ }^{12}$ compared several upper limb anthropometric

20 and range of motion measures taken directly from photographs from a sample of 9 children with

21 unilateral partial hand reductions. The authors found no significant mean difference between the

22 measures taken directly over the arms and those taken from photographs, suggesting the potential

23 development of remote fitting procedures for upper limb 3D printed prostheses. However, as 
1 reported by Diment, et al., ${ }^{13}$ there is a need to assess the efficacy and effectiveness, as well as the

2 clinical outcomes for remote fitting procedures of upper limb 3D printed prostheses.

3 Despite great efforts by nonprofit organizations, such as LN-4, E-nable, and others, a

4 number of individuals with upper limb deficiencies from developing countries are still not being

5 fitted with any type of prosthesis. There is a critical need for practical, customized, aesthetically

6 appealing, low-cost upper limb prostheses that can be fitted remotely. Thus, the purpose of the

7 current investigation is to described a remote fitting procedure for upper limb 3D printed

8 prostheses and assess patient satisfaction and comfort with 3D printed prostheses fitted remotely.

9 We hypothesized that there will be an acceptable level of patient satisfaction and comfort after 4

10 weeks of using an upper limb 3D printed prosthesis fitted remotely. Our hypothesis is based on a

11 previous investigation ${ }^{12}$ that found no significant mean difference between the measures taken

12 directly over the arms and those taken from photographs, suggesting the potential development of

13 remote fitting procedures for upper limb 3D printed prostheses.

\section{Methods}

16 A qualitative study using content and score analysis was used to describe patient satisfaction after

174 weeks of using an upper limb 3D printed prosthesis fitted remotely. Research participants

18 provided photographs following a template provided by our research team (Fig. 1A, B, C, D).

19 Several anthropometric measurements were extracted from the photographs to determine lengths

20 and widths of different segments of the upper limbs. For the wrist-driven 3D printed partial hand

21 prosthesis, additional photographs of wrist extension and flexion were requested to quantify active

22 range of motion. The anthropometric measurements extracted from photographs were used to scale

23 and modify the CAD model of each upper limb prosthesis using a methodology previously 
1 validated by our research team. ${ }^{11}$ After the prosthesis was manufactured it was sent to the research

2 participants via The United States Postal Service with detailed instructions of prosthetic use and

3 care. After 4 weeks of use, the research participants or family members of pediatric patients were

4 asked to complete several surveys and questionnaires.

5 Study Population

6 Six children (three girls and three boys, 6 to 16 years of age) and 2 adults (males of 25 and 59

7 years of age) with congenital $(n=7)$ and acquired $(n=1)$ upper limb loss participated in this study.

8 All participants were remotely fitted with a wrist-driven 3D printed partial hand prosthesis $(n=6)$

9 and an elbow-driven 3D printed trans-radial prosthesis $(\mathrm{n}=2$; Table 1$)$.

10 Table 1 here

11 Inclusion criteria for all participants included individuals from 6 to 80 years of age with

12 unilateral upper-limb reductions involving the hand and forearm, and range of motion for the wrist 13 and elbow joints greater than 20 degrees.

14 Exclusion criteria included presence of an upper extremity injury within the past month 15 and any medical conditions that would contraindicate the use of the transitional prosthesis, such 16 as skin abrasions and musculoskeletal injuries.

All subjects completed a medical history questionnaire. All study participants were 18 informed about the study and parents or guardians signed a parental permission form. For children 19 ages 6 to 10 years, an assent was explained by the corresponding author and signed by the children 20 and their parents. For adults a consent form was explained and signed. The study was approved by 21 the University of Nebraska Medical Center Institutional Review Board.

Two weeks after receiving their pictures, research participants were provided with a 3D 23 printed prosthesis. Detailed safety guidelines were given to the parents regarding the use and care 
1 of the prosthesis. After 4 weeks of using the 3D printed prostheses, participants completed a series

2 of surveys including the Orthotics Prosthetics Users' Survey (OPUS), Quebec User Evaluation of

3 Satisfaction with Assistive Technology (QUEST 2.0), and a general survey developed by our

4 research team. The general survey was developed to estimate the impact of prosthetic devices on

5 elements related to quality of life, daily usage, and types of activities performed. This survey has

6 not been statistically validated, but provides useful information related to daily usage.

\section{Procedures}

$9 \quad$ Remote Prosthetic Fitting Procedures

10 Anthropometric Measurements: Several range of motion and anthropometric measures were 11 extracted from the template photographs using the image editing tool available in Autodesk Fusion 12360 (Fusion 360, Autodesk, Inc., San Rafael, CA, USA). To measure wrist range of motion, 13 participants were requested to actively and maximally extend (Fig. 1A) and flex (Fig. 1B) at the 14 wrist joint. The range of motion measurements for the partial hands included; range of motion of 15 the wrists extension (Fig. 1A1 and A2) and flexion (Fig. 1B1 and B2).

16 Figure 1 here

17 The anthropometric measurements for the partial hands included; hand length (tip of the 18 middle finger to center of the wrist joint for the non-affected and affected hands, Fig. 1C1and C5), 19 palm width (widest region of the palm above the base of the thumb, Fig. 1C2 and C6), forearm 20 length (center of the wrist joint to center of the elbow joint, Fig. 1C3 and C7) and forearm width 21 at three-fourths (width of the forearm at proximal three-fourths of the length of the forearm 22 proximal to the wrist, Fig. 1C4 and C8). In addition, several reference lines were drawn over the 
1 participant's non-affected and affected hands connecting the wrist and elbow joints (Fig. 1C and 2 D, red lines).

Range of motion measurements for the elbow joint were not required for patients with

4 congenital or acquired trans-radial reductions, due to the infrequency of a reduced range of motion

5 at the elbow joint in individuals with trans-radial reductions. Anthropometric measurements of the

6 elbow-driven 3D printed trans-radial prostheses included; hand length (tip of the middle finger to

7 center of the wrist joint; Figure 1D1), palm width (widest region of the palm above the base of the

8 thumb, Fig. 1D2), forearm length (center of the wrist joint to center of the elbow joint of the non-

9 affected and affected arms, Fig. 1D3 and D7), forearm width at three-fourths (width of the forearm

10 at proximal three-fourths of the length of the forearm proximal to the wrist joint of the non-affected

11 and affected arms, Fig. 1D4 and D8), arm lengths (Fig. 1D5 and D9) and arm widths (Fig. 1 D6

12 and D10).

All measurements extracted from the photographs of the affected and non-affected arms

14 were used to fit and scale all 3D printed prostheses (Fig. 2). The measurements from the non-

15 affected hand were used to approach limb symmetry. The main aspects of our digital fitting

16 procedures consisted of properly scaling the digital design of the prosthesis to the dimensions of:

17 1) the patient's residual limb for generating an appropriately sized socket, and 2) the non-affected

18 limb to approximate limbs length and overall symmetry. Our parametric prosthetic design allows

19 standardization of the size of certain structures, such as the wrist mechanism of the 3D printed

20 trans-radial prosthesis and forearm section of the 3D printed partial hand prosthesis. Finally, the

21 remote fitting procedures were inspected by a certified prosthetist by superimposing the prosthetic

22 design over a 2D image (Fig. 2). This superimposition was also used for patient and their clinician

23 approval. The prosthetic socket was integrated on the inner side of the palm for the partial hand 
1 prosthesis and forearm compartment for the arm prosthesis. A neoprene mitten with a

2 thermoplastic shell was manually constructed and secured to the palm of the partial hand

3 prosthesis. For the elbow-driven trans-radial prosthesis, adhesive foam padding was used as

4 needed to improve fitting and comfort of the integrated forearm socket.

5 After the digital fitting had been performed by a technician under the supervision and approval of

6 a clinical team, the design was exported as a stereolithographic file and 3D printed using desktop

7 and semi-industrial 3D printers.

8 Figure 2 here

9 3D-Printed Partial Hand Prosthesis Characteristics

A modified version of the 3D-printed transitional hand prosthesis named Cyborg Beast ${ }^{11}$

11 (Fig. 3) was used in the study. The new version named Cyborg Beast 2, was designed using the

12 modeling software Autodesk Fusion 360 (Fusion 360, Autodesk, Inc., San Rafael, CA, USA) and

13 manufactured in the 3D Printed Prosthetic Orthotic \& Assistive Devices Laboratory located in the

14 Biomechanics Research Building of the University of Nebraska at Omaha. The 3D printers used

15 for the manufacturing process included a combination of desktop and industrial 3D printers

16 (Ultimaker 2, Ultimaker B.V., Geldermalsen, The Netherlands and Uprint SE Plus by Stratasys,

17 Minnesota, USA).

The plastic pins to secure all the various components of the prosthesis, fingers, thumb,

19 palm, socket, forearm brace, and leveraging structure were made of polylactic acid which has

20 properties similar to thermoplastic that facilitate post manufacturing adjustments. Elastic cords

21 placed inside the dorsal aspect of the fingers provided passive finger extension. Finger flexion was

22 driven by non-elastic cords along the palmar surface of each finger and was activated through $20^{\circ}$

23 of wrist flexion. The result was a composite fist (flexing the fingers towards the palm) for gross 
1 grasp. The finger and thumb were oriented in opposition to facilitate cylindrical grasp and tip

2 pinch. A BOA dial tensioner system (Mid power reel M3, BOA Technology Inc., Denver,

3 Colorado) was used to regulate the tension of the cables controlling the finger flexion. A brace

4 leverage structure was included in the proximal aspect of the forearm to increase torque

5 development and stability. A thermoplastic socket embedded in the palmar aspect of the hand

6 prosthesis was added to facilitate fitting of the device. The hand prosthesis was customized to each

7 individual limb size and aesthetic requirements, such as colors and specific designs (Fig. 3).

$8 \quad$ Figure 3 here

9 3D-Printed Arm Prosthesis Characteristics

10 The hand had 5 fingers with 2 degrees of freedom (Fig. 4). The finger and thumb were oriented in 11 opposition to facilitate cylindrical grasp and tip pinch. Silicone finger pads were added to provide 12 increased friction for grasping activities. A rotation mechanism placed in the wrist allowed for full 13 pronation and supination, and a pivot system with internal components allowed for wrist rotation 14 without twisting the line activating the flexion of the fingers. The rotation mechanism of the wrist 15 consisted of an inner circular disc/shaft with a center opening (Fig. 4B). A circle of embedded 16 magnets with matching polarity was placed around the disc. A bi-valve circular sleeve with 17 embedded magnets aligned to match the disc magnets, was placed over the disc. The magnets were 18 placed with opposing polarity to assure mutual attraction. The disc and sleeve rotated 19 independently and fixed in various positions by the attraction of the magnets. The magnets were 20 sealed in a protective sleeve for safety. Elbow flexion and extension could be performed using a 21 simple hinge mechanism. A BOA dial tensioner system allowed for the regulation of the tension 22 of the cables controlling the finger flexion. A Velcro strap secured the prosthesis to the arm and 23 harnessing was not needed for suspension. 
1

2

3

4

6

7

12

Figure 4 here

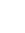

\section{$3 D$ Printing Specifications}

The 3D printed prostheses were manufactured using desktop and industrial 3D printers

(Ultimaker 2, Ultimaker B.V., Geldermalsen, The Netherlands and Uprint SE Plus by Stratasys,

Minnesota, USA). The materials used for printing the prosthetic hand were Raptor polylactic acid.

All parts were printed at $40 \%$ infill (hexagon pattern for desktop, crosshatch for industrial), 60$100 \mathrm{~mm} / \mathrm{s}$ print speed, $150-200 \mathrm{~mm} / \mathrm{s}$ travel speed, $70^{\circ} \mathrm{C}$ heated chamber for acrylonitrile butadiene styrene $\left(50^{\circ} \mathrm{C}\right.$ heated bed for plolylactic acid), 0.15-.25 $\mathrm{mm}$ layer height, and $1 \mathrm{~mm}$ shell thickness. Rafts and supports were used to 3D print the palm and other delicate components. Production time ranged from 4 to 10 hours to 3D print and fully assemble the prostheses.

\section{Prosthesis Use, Fitting, and Satisfaction Surveys}

Prosthesis use was assessed using the Quebec User Evaluation of Satisfaction with assistive Technology (QUEST 2.0) ${ }^{14}$ which includes items related to assistive device satisfaction, wear, and use. Prosthesis use was also assessed with the Orthotics Prosthetics Users Survey (OPUS) which includes items related to weight, comfort, ease of use and function. ${ }^{15}$ The QUEST 2.0 is a standardized self-report questionnaire that is designed to evaluate how satisfied patients are with their assistive devices and the related services they experienced. The survey consisted of 12 satisfaction items (dimensions, weight, adjustments, safety, durability, easy to use, comfort, effectiveness, service delivery, repairs, professional services, follow-up services) where each item receives a rating from 1 to 5, with 1 being 'not satisfied at all' and 5 being 'very satisfied'. The QUEST questionnaire also includes a ranking of importance for the 12 satisfaction items. Patients 
1 or parents were asked to select 3 items that they consider to be the most important to them (Figure

$25)$.

The OPUS is a self-report questionnaire consisting of several items related to satisfaction

4 and function with the prosthetic device and services. ${ }^{15}$ The OPUS module for upper extremity

5 functional status, which measure the person's perceived ability to perform activities involving the

6 extremities; client satisfaction with device; and client satisfaction with services was used to assess

7 different satisfaction and function items. To assess general aspect of prosthetic use, patients and

8 parents were asked to complete general survey developed by our research team to include the type

9 of activities performed as well as the estimated daily use of the prosthesis.

10 Data Analysis

11 Descriptive statistics (Mean \pm Standard Deviation) were calculated and summarized.

\section{Results}

14 The characteristics of the research participants and reported prosthesis use are summarized in table

15 1. The descriptive information for the QUEST questionnaire (device and service satisfaction) are

16 summarized in Table 2 and patient importance ratings for the satisfaction items are illustrated in

17 Figure 5. The OPUS satisfaction items are summarized in Table 3 and 4. The results of the general 18 prosthetic survey developed by our research team are described in Table 5.

19 Table 1 to 5 here

20 Figure 5 here

\section{Discussion}

The main findings reported in the present study suggest that there is an acceptable level of 23 patient satisfaction with the 3D printed prostheses fitted remotely. The research participants 
1 reported a score of $3.92 \pm 0.50$ (closer to the statement "quite satisfied") for the device satisfaction

2 section of the QUEST questionnaire (Table 2). This acceptable level of satisfaction of our research

3 participant reported in the QUEST was confirmed by the agreement scores of the OPUS items

4 related to prosthetic fitting (My prosthesis fits well $=4.13 \pm 0.50)$ and comfort (My prosthesis is

5 comfortable throughout the day $=3.57 \pm 0.98)$. Furthermore, the comfort level rating in the general

6 prosthetic survey resulted in a score of $3.75 \pm 0.70$ (closer to the statement "the prosthetic device

7 feels comfortable") confirming the results of the QUEST and OPUS.

There is limited literature describing remote fitting procedures for upper limb 3D printed

9 prosthesis. ${ }^{11}$ Zuniga et al., ${ }^{11}$ described a remote fitting methodology for 3D printed upper limb prostheses and compared anthropometric measurements taken directly over the patients upper limb

11 from measurements taken from photographs. The authors found no significant mean difference

12 between the measures taken directly over the arms and those taken from photographs in a sample

13 of nine children with partial hand reductions. Furthermore, a recent systematic review by Diment,

14 et al., ${ }^{13}$ indicated the efficacy or effectiveness of upper limb 3D printed prostheses has not been

15 properly assessed. The authors ${ }^{13}$ emphasized the need to assess clinical outcomes for remote fitting

16 procedures of upper limb 3D printed prostheses. However, there are no studies examining the

17 patient satisfaction with remote prosthetic fitting and only a few studies that have addressed patient

18 satisfaction with standard prostheses and fitting. A previous investigation by Routhier et al., ${ }^{16}$

19 examined the satisfaction level of 10 pediatric research subjects ( 7 girls and 3 boys from 2.5 to

2016.0 years of age) with partial hand or below the wrist amputations $(n=3)$, as well as trans-radial

$21(n=6)$ and trans-humeral amputations $(n=1)$. All research subjects were fitted with myoelectric

22 prostheses using the VASI $(n=9)$ and Steeper $(n=1)$ electric hands as terminal devices. ${ }^{16}$ The

23 standard fitting procedures were performed at the Quebec Rehabilitation Institute in Canada and 
1 the clinical team used an early version of the QUEST questionnaire to assess patient satisfaction.

2 In general, the study found that $60 \%$ of the participants were satisfied with the prosthesis.

3 Specifically, the authors reported that 6 participants were "very satisfied or satisfied", 3

4 participants were "somewhat satisfied" or "rather unsatisfied" and 1 participant was "not satisfied 5 at all". ${ }^{16}$

In the present investigation, we found that for the device subscale of the QUEST only

$7 \quad 37.5 \%$ of the participants were satisfied with the device fitted remotely. Specifically, 3 participants

8 were "satisfied" (scores $=4.63,4.5$, and 4.5) and 5 participants were "more or less satisfied" (3.5,

$93.88,3.63,3.25$, and 3.75). However, the category that is most relevant for prosthetic fitting, comfort, $50 \%$ of the subjects were satisfied with the device fitted remotely. Specifically, two

11 participants were "very satisfied", two participants were "quite satisfied", and 4 participants were

12 "more or less satisfied" with the overall comfort of the prosthesis fitted remotely. These findings

13 are similar to those reported by Routhier et al., ${ }^{16}$ where the authors reported $50 \%$ satisfaction with

14 the comfort of an upper-limb myoelectric prosthesis fitted using standard procedures at the Quebec

15 Rehabilitation Institute. It should be noted, however, that obtaining a 50\% satisfaction of comfort

16 using a myoelectric prosthesis is significantly more difficult than for body-powered prosthesis due

17 to the extra weight of the electronic components of myoelectric devices.

A previous investigation by Ghoseiri \& Bahramian $^{17}$ evaluated the user satisfaction with orthotic and prosthetic devices and services using the OPUS in a sample of 293 patients. Using 20 standard fitting procedures, patients using lower limb orthoses $(n=178)$, spinal orthoses $(n=36)$, 21 upper limb orthoses $(n=40)$, lower limb orthoses $(n=34)$ and upper limb prostheses or partial hands $22(n=5)$ completed the OPUS survey. Overall, the authors ${ }^{17}$ found that patients were unsatisfied with 23 their devices with only $17 \%$ satisfaction rate. These results are much lower than those found by 
1 Bosmans et al. ${ }^{18}$ and Routhier et al. ${ }^{16}$ reporting $78 \%$ and $80 \%$ satisfaction with prostheses and

2 orthoses using similar fitting procedures. As suggested by Ghoseiri \& Bahramian ${ }^{17}$ the type of

3 reduction, deformity, injury and overall morphology of the residual limb may affect the results of

4 satisfaction and comfort with their prostheses or orthoses. Similarly, in the current investigation,

5 five of the eight research participants had partial hand reductions with different anatomical and

6 morphological features of the affected limb (Table 1). Thus, the challenges of fitting partial hand

7 prostheses for individuals with different anatomical and morphological reductions may have

8 prevented our research participants from been fully satisfied with the comfort of the prosthesis

9 (Tables 2 and 3). The overall acceptable level of patient satisfaction with the 3D printed prostheses

10 fitted remotely, however, was likely due to a combination of the versatility of fitting these devices

11 using an editable digital file and the thermoforming properties of the 3D printing filament that

12 allows post-processing adjustments of the sockets. Furthermore, for two of the research

13 participants with partial hand reductions (Subject 5 and 6) a custom neoprene socket was

14 developed to improve comfort. The three research participants with trans-radial reductions had

15 similar residual morphology of the residual limb allowing for the integration of the socket inside

16 of the forearm compartment. The 3D printed arm prosthesis was scaled to approximate the non-

17 affected arm of the patient for symmetry purposes. The patients and their supervising clinician

18 were instructed to use adhesive foam padding as needed to improve fitting and comfort of the 19 forearm socket.

20 In the current study, the satisfaction items of the OPUS agreement scores ranged from 3.67

$21 \pm 1.03$ (My prosthesis is durable) to $4.57 \pm 0.92$ (The weight of my prosthesis is manageable; Table

22 3). Patients tended to agree with the statement "My prosthesis fits well" ( $4.14 \pm 0.53$ Table 3$).$

23 Furthermore, the prosthetic function module of the OPUS indicated that the majority of the 
1 research participants found that "drinking from a paper cup" $(4.00 \pm 1.20)$ and "putting on and

2 taking off the prosthesis" ( $4.5 \pm 0.84)$ was easy (Table 4$)$. For other functional items, such as "dial

3 a touch tone phone" $(3.0 \pm 2.0)$ or "stir in a bowl" $(3.50 \pm 1.52)$ the research participants reported

4 that the activity was slightly difficult (Table 4).

Among the many challenges experienced by adult and pediatric prosthetic users, as well as

6 their families, is the distance from the patient residence and the rehabilitation center. ${ }^{16}$ Previous

7 investigations and reports ${ }^{19}, 20$ indicated that a number of children and adults with upper limb loss

8 from developing countries and rural areas have many challenges accessing prosthetic services.

9 These challenges are due to many factors, such as the lack of trained technicians able to provide

10 these services and a local shortage of the necessary componentry for the production of upper limb

11 prostheses. ${ }^{19,20}$ The remote fitting procedures presented in the current investigation can be helpful

12 by allowing health care professionals from developed countries to remotely fit and manufacture

13 the devices and send them to the medical team supervising the patient. The remote fitting

14 procedures described in the present study require minimal anthropometric measurements of the

15 upper limbs for proper scaling and fitting. Most fitting procedures required for prosthetic hands

16 include wrap casting using plaster bandages placed over the affected limb. ${ }^{21}$ More recently, 3D

17 scanning has also been used for the development of different types of prosthetic sockets for upper-

18 limb and lower-limb prostheses. ${ }^{22-24}$ Casting procedures require the physical presence of the

19 individual needing the prosthetic hand and the health care professional in the same physical

20 location, which may not be possible for patients living in rural or isolated areas. 3D scanning

21 procedures require sophisticated equipment and technical knowledge to perform the

22 measurements. Furthermore, both techniques require the patient to visit the health care facilities

23 for proper fitting procedures. The results from the present investigation describe a novel and simple 
1 remote fitting procedure for 3D-printed upper-limb prosthesis for children and adults with upper-

2 limb loss. The proposed distance-fitting procedures have the potential of making 3D printed

3 prostheses accessible to a large number of individuals in rural or isolated areas. These procedures,

4 however, must be performed with caution, since inaccurate scaling or significant errors in the 5 measurements could affect the function and fitting of the 3D printed prosthesis.

6 The potential limitations of the present investigation include the individual differences of 7 our research participants at multiple levels including age, gender, daily prosthetic use and etiology 8 of the upper limb reduction, as well as the different anatomical and morphological features of the 9 affected limb (trans-radial versus partial hands; Table 1). This inter-subject variability and the 10 qualitative nature of self-reported questionnaires and surveys may have affected the satisfaction 11 and function scores presented in the current investigation. Furthermore, family members of the 12 four youngest research participants (6 to 10 years of age) helped respond to the questions of the 13 surveys; therefore, some of the responses may not be accurate. For this reason, we asked all of our 14 research participants to complete three different surveys (i.e., QUEST, OPUS, and a general 15 prosthetic survey) to confirm their responses.

16 In conclusion, the ability to fit an upper-limb prosthesis remotely, represents a promising 17 methodology to fit upper-limb 3D printed prostheses for patients from developing countries or 18 rural areas. The increasing availability of smartphones and other digital devices makes it possible 19 to obtain photographs from patients located in rural areas that have little or no access to trained 20 technicians. These photographs along with the cost-effective desktop 3D printers allows for the 21 extraction of the anthropometric measurements required for the development of a 3D printed upper 22 limb prosthesis remotely. 
1

12

\section{Clinical Messages}

- Only 1 in 9,400 children and adults are considered for prosthetic fitting due to the complexity of the reduction and fitting procedures.

- $35 \%$ to $58 \%$ of children with access to prostheses reject them due to excessive weight, lack of functional use, discomfort, and low visual appeal of the device

- 3D printing technology offers the unique possibility of fitting, designing, and manufacturing low-cost and customized upper-limb 3D printed prostheses remotely.

- There is an acceptable level of patient satisfaction with the $3 \mathrm{D}$ printed prostheses fitted remotely.

- The ability to fit an upper-limb prosthesis remotely, represents a promising methodology to fit upper-limb 3D printed prostheses for patients from developing countries or rural areas.

\section{Acknowledgements}

We would like to thank the adults and children for participating in our research.

Thanks to the students working in the 3D Printed Prosthetic, Orthotic \& Assistive Devices Laboratory at the Department of Biomechanics who helped with data collection and device manufacturing.

9 Disclosure statement

Dr. Jorge M. Zuniga, Jean Peck, and Rakesh Srivastava lead the research team that designed the $13 \mathrm{D}$ printed prostheses and other devices discussed in this article. The rest of the researchers declare 2 no competing interests. 


\section{Funding}

2 Funding for this study was provided by the University of Nebraska Collaboration Grant, the Center

3 for Research in Human Movement Variability of the University of Nebraska Omaha and the NIH 4 (P20GM109090). 


\section{References}

1. CDC. Facts about Upper and Lower Limb Reduction Defects. 2014.

2. Giele $\mathrm{H}$, Giele $\mathrm{C}$, Bower $\mathrm{C}$ and Allison $\mathrm{M}$. The incidence and epidemiology of congenital upper limb anomalies: a total population study. The Journal of hand surgery. 2001; 26: 628-34.

3. Canfield MA, Honein MA, Yuskiv N, et al. National estimates and race/ethnic-specific variation of selected birth defects in the United States, 1999-2001. Birth defects research Part A, Clinical and molecular teratology. 2006; 76: 747-56.

4. Resnik L, Meucci MR, Lieberman-Klinger S, et al. Advanced upper limb prosthetic devices: implications for upper limb prosthetic rehabilitation. Archives of physical medicine and rehabilitation. 2012; 93: 710-7.

5. Parker SE, Mai CT, Canfield MA, et al. Updated National Birth Prevalence estimates for selected birth defects in the United States, 2004-2006. Birth defects research Part A, Clinical and molecular teratology. 2010; 88: 1008-16.

6. Resnik L. Development and testing of new upper-limb prosthetic devices: research designs for usability testing. Journal of rehabilitation research and development. 2011; 48: 697-706.

7. James MA, Bagley AM, Brasington K, Lutz C, McConnell S and Molitor F. Impact of prostheses on function and quality of life for children with unilateral congenital below-the-elbow deficiency. The Journal of bone and joint surgery. 2006; 88: 2356-65.

8. Hadders-Algra M, Reinders-Messelink HA, Huizing K, van den Berg R, van der Sluis CK and Maathuis CG. Use and functioning of the affected limb in children with unilateral congenital below-elbow deficiency during infancy and preschool age: a longitudinal observational multiple case study. Early human development. 2013; 89: 49-54.

9. Huizing K, Reinders-Messelink H, Maathuis C, Hadders-Algra M and van der Sluis CK. Age at first prosthetic fitting and later functional outcome in children and young adults with unilateral congenital below-elbow deficiency: a cross-sectional study. Prosthetics and orthotics international. 2010; 34: 166-74.

10. Biddiss EA and Chau TT. Upper limb prosthesis use and abandonment: a survey of the last 25 years. Prosthetics and orthotics international. 2007; 31: 236-57.

11. Zuniga JM, Katsavelis D, Peck J, et al. Cyborg beast: a low-cost 3d-printed prosthetic hand for children with upper-limb differences. BMC research notes. 2015; 8: 10.

12. Zuniga JM, Peck J, Srivastava R, Katsavelis D and Carson A. An Open Source 3D-Printed Transitional Hand Prosthesis for Children. JPO: Journal of Prosthetics and Orthotics. 2016; 28: 103-8.

13. Diment LE, Thompson MS and Bergmann JH. Three-dimensional printed upper-limb prostheses lack randomised controlled trials: A systematic review. Prosthetics and orthotics international. 2018; 42: 7-13.

14. Demers L, Weiss-Lambrou R and Ska B. Item analysis of the Quebec User Evaluation of Satisfaction with Assistive Technology (QUEST). Assistive technology : the official journal of RESNA. 2000; 12: 96-105.

15. Heinemann AW, Bode RK and O'Reilly C. Development and measurement properties of the Orthotics and Prosthetics Users' Survey (OPUS): a comprehensive set of clinical outcome instruments. Prosthetics and orthotics international. 2003; 27: 191-206.

16. Routhier F, Vincent C, Morissette MJ and Desaulniers L. Clinical results of an investigation of paediatric upper limb myoelectric prosthesis fitting at the Quebec Rehabilitation Institute. Prosthetics and orthotics international. 2001; 25: 119-31. 
1 17. Ghoseiri $\mathrm{K}$ and Bahramian $\mathrm{H}$. User satisfaction with orthotic and prosthetic devices and 2 services of a single clinic. Disability and rehabilitation. 2012; 34: 1328-32.

3 18. Bosmans J, Geertzen J and Dijkstra PU. Consumer satisfaction with the services of 4 prosthetics and orthotics facilities. Prosthetics and orthotics international. 2009; 33: 69-77.

5 19. Cummings D. Prosthetics in the developing world: a review of the literature. Prosthetics 6 and orthotics international. 1996; 20: 51-60.

7 20. ISPO consensus conference on appropriate orthopaedic technology for low-income 8 countries: conclusions and recommendations. International Society for Prosthetics and Orthotics. 9 Prosthetics and orthotics international. 2001; 25: 168-70.

10 21. Bray JJ and University of California Los Angeles. Prosthetics-Orthotics Education 11 Program. Prosthetic principles, upper extremity amputations : fabrication and fitting principles. 12 Los Angeles: Prosthetics-Orthotics Education Program, Division of Orthopedic Surgery, 13 University of California, Los Angeles, 1970.

14 22. Rengier F, Mehndiratta A, von Tengg-Kobligk H, et al. 3D printing based on imaging data: 15 review of medical applications. International journal of computer assisted radiology and surgery. $162010 ; 5: 335-41$.

17 23. Vera C, Barrero C, Shockley W, Rothenberger S, Minsley G and Drago C. Prosthetic 18 Reconstruction of a Patient with an Acquired Nasal Defect Using Extraoral Implants and a 19 CAD/CAM Copy-Milled Bar. Journal of prosthodontics : official journal of the American College 20 of Prosthodontists. 2014.

21 24. Dombroski CE, Balsdon ME and Froats A. The use of a low cost 3D scanning and printing 22 tool in the manufacture of custom-made foot orthoses: a preliminary study. BMC research notes. $232014 ; 7: 443$. 
1 Table 1: Characteristics of research participants $(n=9)$

\begin{tabular}{ccccccc}
\hline ID & Gender & $\begin{array}{c}\text { Age } \\
\text { (Years) }\end{array}$ & Reported Prosthesis Use & $\begin{array}{c}\text { Amputation } \\
\text { Level }\end{array}$ & $\begin{array}{c}\text { Affected } \\
\text { Side }\end{array}$ & $\begin{array}{c}\text { Ability } \\
\text { to Pinch }\end{array}$ \\
\hline $\mathbf{1}$ & M & 59 & 6+ hours per day & *Trans-Radial & Right & No \\
$\mathbf{2}$ & M & 25 & 6+ hours per day & Partial Hand & Right & No \\
$\mathbf{3}$ & M & 14 & Between 1-2 hours per day & Partial Hand & Right & No \\
$\mathbf{4}$ & M & 10 & 10 minutes, 5 times per week & Partial Hand & Left & No \\
$\mathbf{5}$ & M & 6 & Only when needed & Trans-Radial & Left & No \\
$\mathbf{6}$ & F & 16 & 1 hour per day & Partial Hand & Left & Some \\
$\mathbf{7}$ & F & 8 & Only when needed & Trans-Radial & Left & No \\
$\mathbf{8}$ & F & 6 & 10 minutes, 5 times per week & Partial Hand & Right & No \\
\hline
\end{tabular}


Table 2. Quebec User Evaluation of Satisfaction with assistive Technology (QUEST) Ratings.

\begin{tabular}{|c|c|c|c|}
\hline $\begin{array}{l}\text { Items } \\
\text { How satisfied are you with: }\end{array}$ & Mean \pm SD & $\begin{array}{c}\text { Minimum } \\
\text { Score } \\
\end{array}$ & $\begin{array}{c}\text { Maximum } \\
\text { Score } \\
\end{array}$ \\
\hline Dimensions (size, height, length, width) & $4.13 \pm 0.99$ & 2 & 5 \\
\hline Weight & $4.50 \pm 0.76$ & 2 & 5 \\
\hline Adjustments (fixing, fastening) & $4.13 \pm 0.83$ & 3 & 5 \\
\hline Safety (secure) & $4.38 \pm 0.52$ & 4 & 5 \\
\hline Durability (endurance, resistance to wear) & $3.13 \pm 0.83$ & 2 & 4 \\
\hline Ease of Use & $4.13 \pm 0.64$ & 3 & 5 \\
\hline Comfort & $3.75 \pm 0.89$ & 3 & 5 \\
\hline $\begin{array}{l}\text { Effectiveness (the degree to } \\
\text { which your device meets your needs) }\end{array}$ & $3.50 \pm 1.31$ & 2 & 5 \\
\hline $\begin{array}{l}\text { Device } \\
\text { Satisfaction } \\
\end{array}$ & $3.95 \pm 0.85$ & 2 & 5 \\
\hline $\begin{array}{l}\text { Services } \\
\text { How satisfied are you with: }\end{array}$ & Mean \pm SD & $\begin{array}{l}\text { Minimum } \\
\text { Score }\end{array}$ & $\begin{array}{l}\text { Maximum } \\
\text { Score }\end{array}$ \\
\hline Service Delivery & $4.25 \pm 0.89$ & 3 & 5 \\
\hline Repairs and Servicing & $4.25 \pm 0.89$ & 3 & 5 \\
\hline Professional Services & $4.75 \pm 0.46$ & 4 & 5 \\
\hline Follow-up Service & $4.75 \pm 0.46$ & 4 & 5 \\
\hline $\begin{array}{c}\text { Service } \\
\text { Satisfaction } \\
\end{array}$ & $4.50 \pm 0.67$ & 3 & 5 \\
\hline $\begin{array}{c}\text { Total } \\
\text { Satisfaction } \\
\end{array}$ & $4.10 \pm 0.49$ & & \\
\hline
\end{tabular}


Table 3. Orthotics Prosthetics Users Survey (OPUS) Frequency Distribution.

\section{OPUS Module 5:}

Raw Scores Raw Score

Satisfaction Items

\begin{tabular}{lllllll}
5 & 4 & 3 & 2 & 1 & 0 & Mean \pm SD \\
\hline & 6 & 1 & 0 & 0 & 0 & $\mathbf{4} .14 \pm 0.53$
\end{tabular}

My prosthesis fits well.

$4.14 \pm 0.53$

The weight of my prosthesis is manageable.

$\begin{array}{lllllll}5 & 1 & 2 & 0 & 0 & 0 & \mathbf{4 . 5 7} \pm \mathbf{0 . 9 2}\end{array}$

My prosthesis is comfortable throughout the day.

$\begin{array}{lllllll}1 & 3 & 1 & 1 & 0 & 2 & \mathbf{3 . 6 7} \pm \mathbf{1 . 0 3}\end{array}$

It is easy to put on my prosthesis.

My prosthesis looks good.

$\begin{array}{lllllll}4 & 2 & 1 & 1 & 0 & 0 & \mathbf{4 . 4 3} \pm \mathbf{1 . 1 3}\end{array}$

My prosthesis is durable.

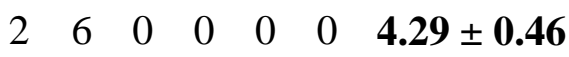

$5=$ Strongly Agree, $4=$ Agree, $3=$ Neither Agree nor Disagree, $2=$ Disagree, $1=$ Strongly Disagree, $0=$ Don't Know. Mean values calculated excluding $0=$ Don't Know raw scores. 
Table 4. Orthotics Prosthetics User Survey (OPUS) Frequency Distribution.

\begin{tabular}{lccccccc}
\hline OPUS Module 5: & \multicolumn{4}{c}{ Raw Scores } & \multicolumn{3}{c}{ Raw Score } \\
Functional Items & 5 & 4 & 3 & 2 & 1 & 0 & Mean \pm SD \\
\hline Drink from a paper cup & 4 & 1 & 2 & 1 & 0 & 0 & $\mathbf{4 . 0 0} \pm \mathbf{1 . 2 0}$ \\
Use fork or spoon & 2 & 2 & 1 & 2 & 0 & 1 & $\mathbf{3 . 5 7} \pm \mathbf{1 . 2 7}$ \\
Pour from a 12 oz. can & 4 & 0 & 1 & 1 & 1 & 1 & $\mathbf{3 . 7 1} \pm \mathbf{1 . 7 0}$ \\
Dial a touch tone phone & 2 & 0 & 1 & 0 & 2 & 1 & $\mathbf{3 . 0} \pm \mathbf{2 . 0}$ \\
Stir in a bowl & 2 & 1 & 2 & 0 & 1 & 0 & $\mathbf{3 . 5 0} \pm \mathbf{1 . 5 2}$ \\
Put on and take off prosthesis & 4 & 1 & 1 & 0 & 0 & 0 & $\mathbf{4 . 5} \pm \mathbf{0 . 8 4}$ \\
\hline
\end{tabular}

5 = Very Easy, 4 = Easy, 3 = Slightly Difficult, 2 = Very Difficult, 1 = Cannot Perform Activity, $0=$ Not Applicable. Mean values calculated excluding $0=$ Not Applicable raw scores . 
Table 5. General Prosthetic Survey.

\begin{tabular}{|c|c|c|c|c|c|}
\hline ID & $\begin{array}{c}\text { In what type of } \\
\text { activities do you } \\
\text { use your prosthetic } \\
\text { device? }\end{array}$ & $\begin{array}{l}\text { Is your current } \\
\text { prosthesis } \\
\text { improving your } \\
\text { quality of life? }\end{array}$ & $\begin{array}{c}\text { Is your current } \\
\text { prosthesis } \\
\text { improving your } \\
\text { self-esteem? }\end{array}$ & $\begin{array}{c}\text { Has your } \\
\text { prosthesis } \\
\text { malfunctioned } \\
\text { or break? }\end{array}$ & $\begin{array}{l}\text { Please rate the } \\
\text { comfort of } \\
\text { your prosthetic } \\
\text { device on a } \\
\text { scale from } 1 \text { to } \\
5^{*} \\
\end{array}$ \\
\hline $\mathbf{1}$ & $\begin{array}{c}\text { Activities at home, } \\
\text { Activities at } \\
\text { school/work, Just for } \\
\text { fun }\end{array}$ & Yes & Yes & Yes & 4 \\
\hline 2 & $\begin{array}{c}\text { Activities at home, } \\
\text { Activities at } \\
\text { school/work }\end{array}$ & No & No & Yes & 4 \\
\hline 3 & $\begin{array}{c}\text { Play, Activities at } \\
\text { home, Activities at } \\
\text { school/work, Just for } \\
\text { fun }\end{array}$ & Somewhat & Yes & Yes & 3 \\
\hline 4 & $\begin{array}{l}\text { Activities at } \\
\text { school/work }\end{array}$ & Somewhat & Somewhat & Yes & 4 \\
\hline 5 & $\begin{array}{c}\text { Activities at } \\
\text { school/work, } \\
\text { Activities at home }\end{array}$ & Somewhat & Somewhat & Yes & 5 \\
\hline 6 & $\begin{array}{c}\text { Activities at home, } \\
\text { Other }\end{array}$ & Yes & Somewhat & No & 3 \\
\hline 7 & $\begin{array}{c}\text { Sports, Activities at } \\
\text { home }\end{array}$ & No & No & No & 3 \\
\hline \multirow[t]{2}{*}{8} & Activities at home & Somewhat & Somewhat & No & 4 \\
\hline & & & & Mean \pm SD & $3.75 \pm 0.70$ \\
\hline
\end{tabular}


1 Figures

2 Figure 1

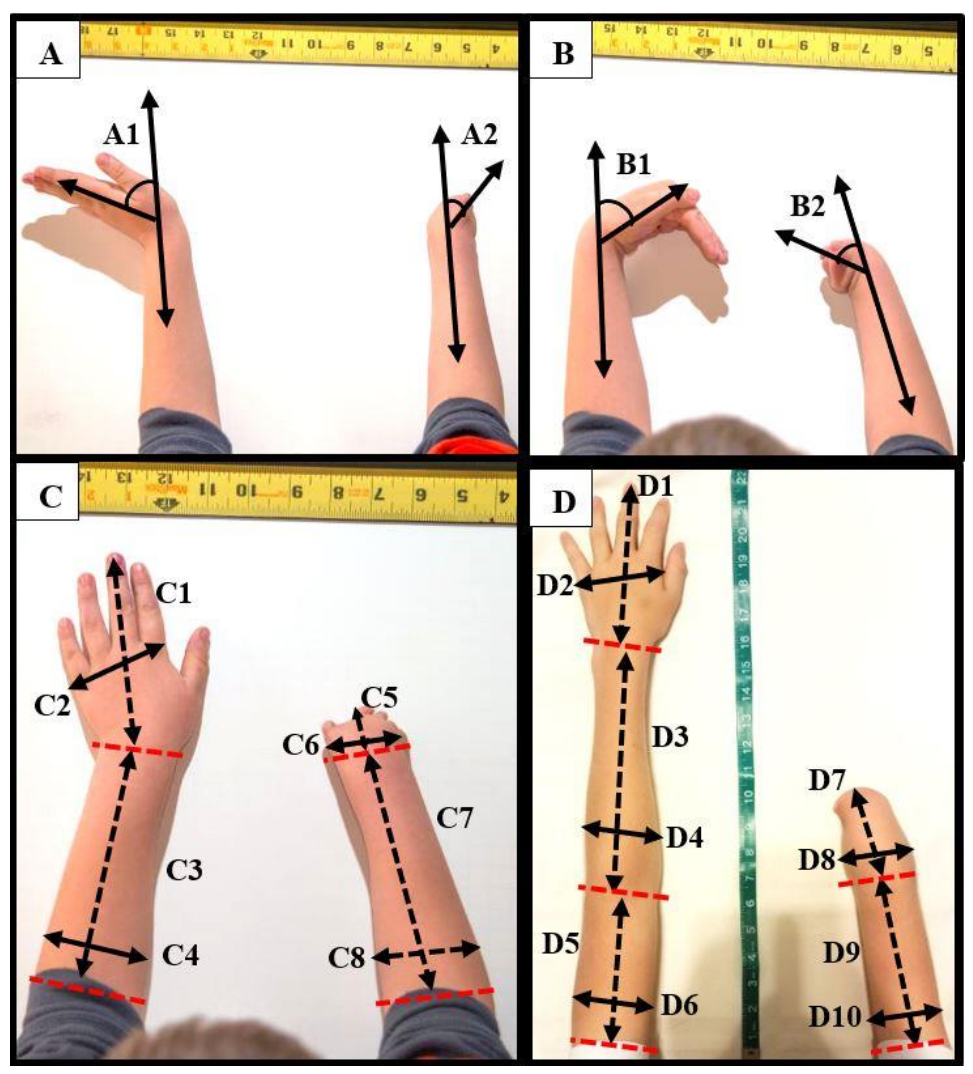


$1 \quad$ Figure 2

2

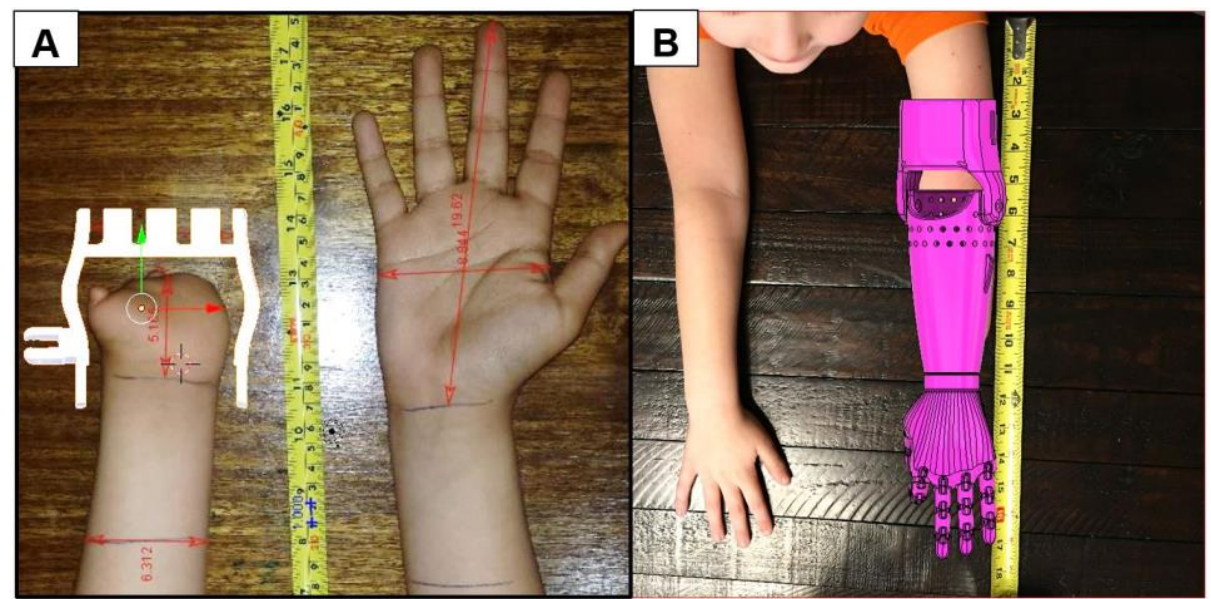


$1 \quad$ Figure 3

2

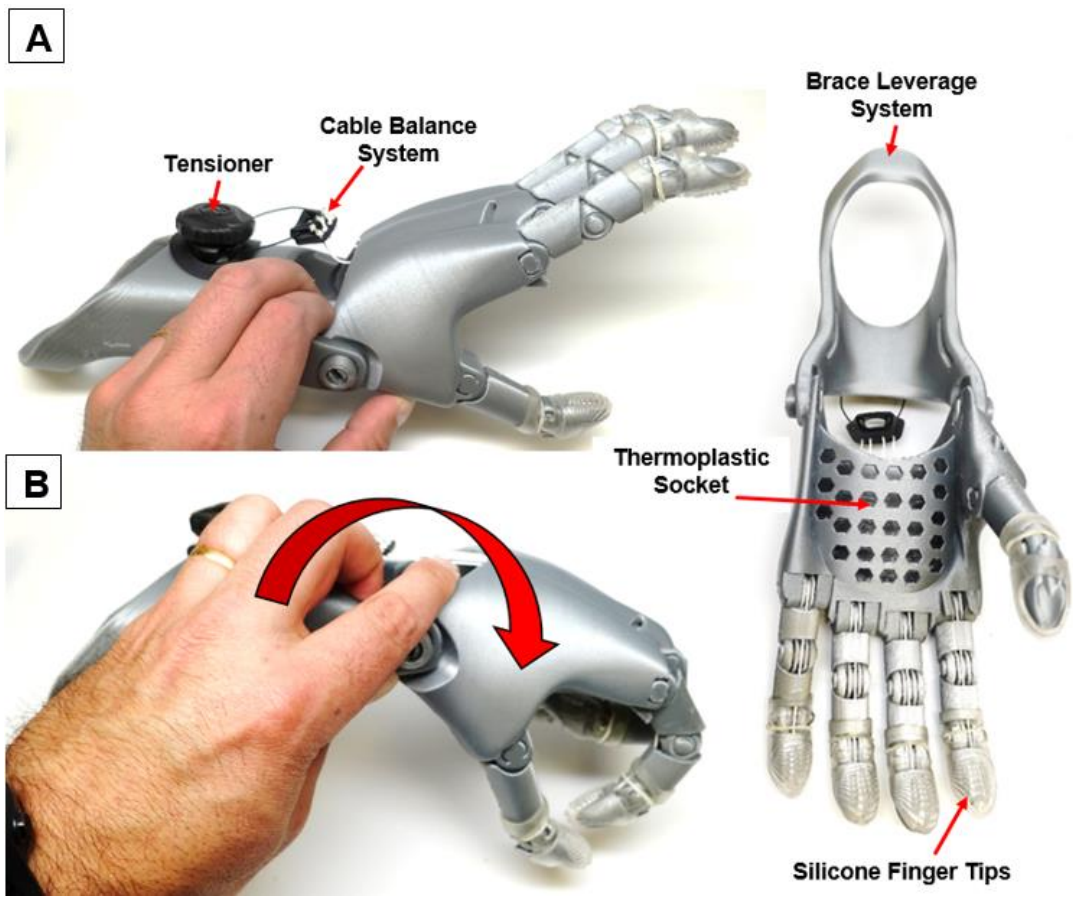


$1 \quad$ Figure 4

2

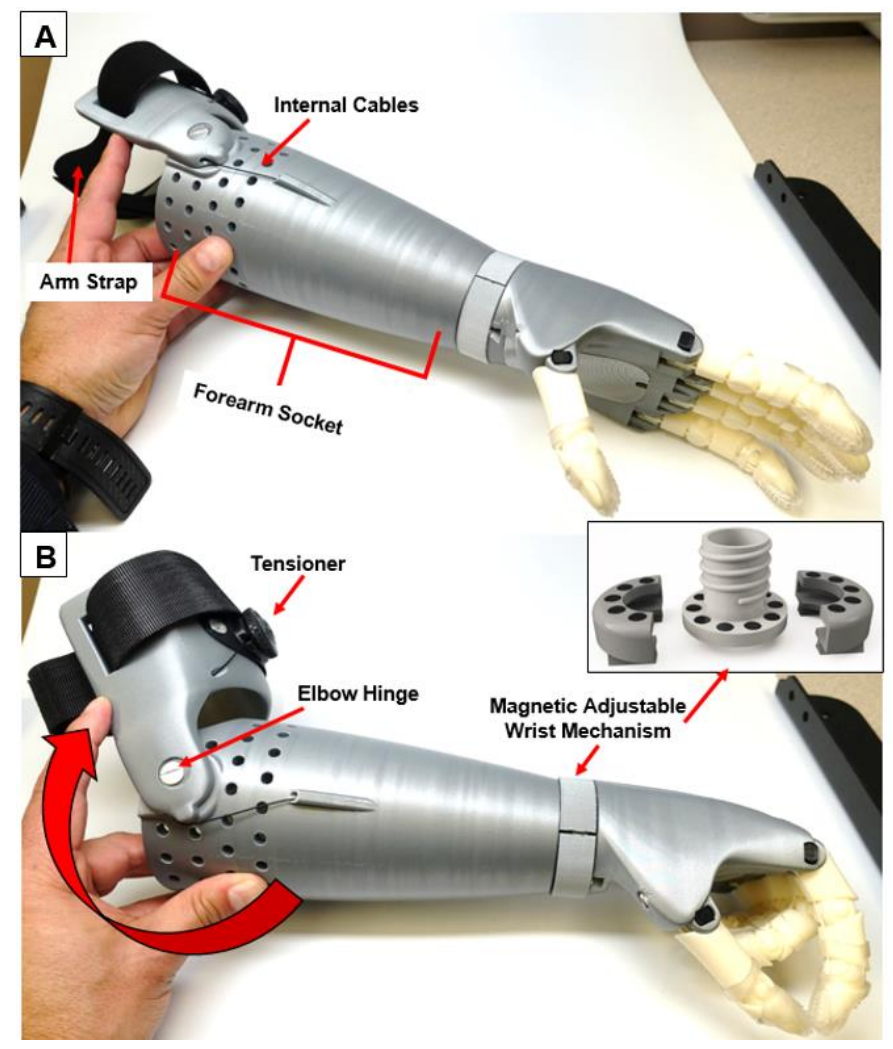


$1 \quad$ Figure 5

\section{Three Most Important Satisfaction Items}

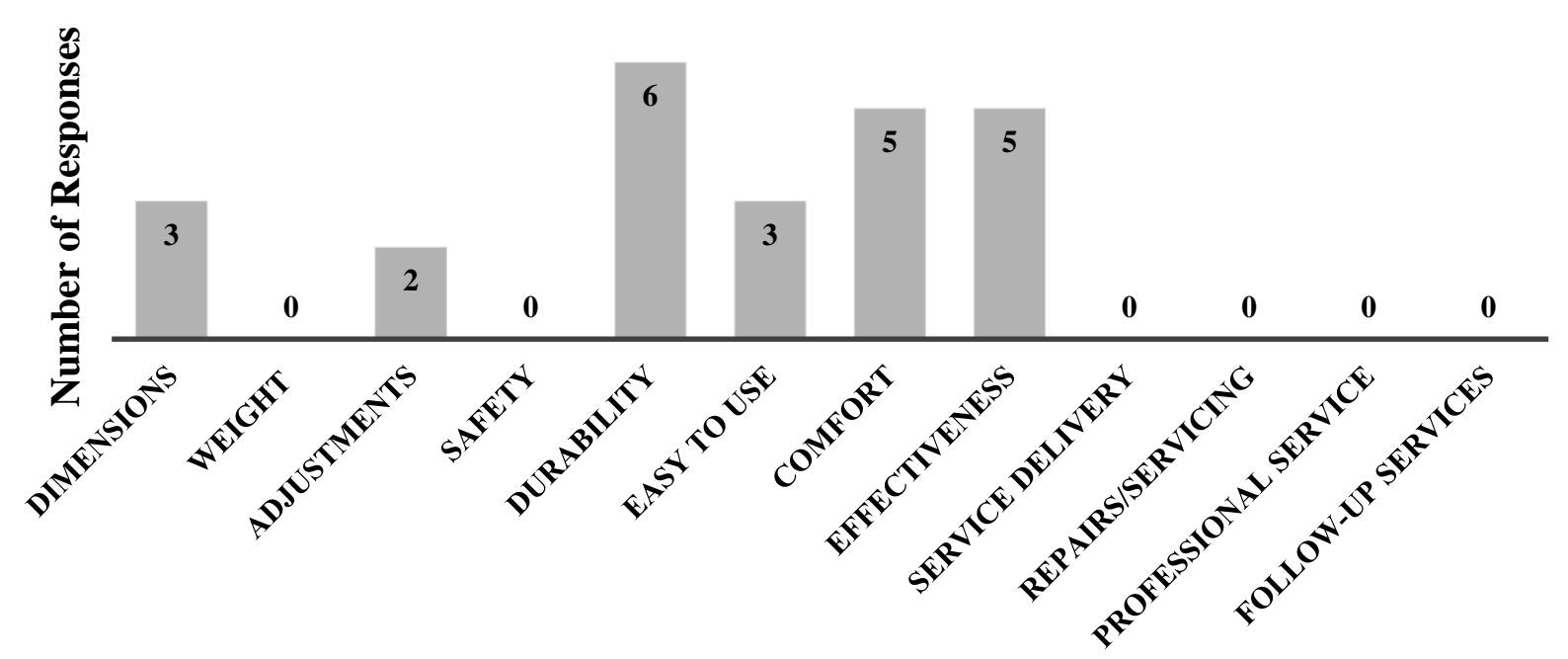

Satisfaction Items 


\section{$1 \quad$ Figure legends}

Figure 2. Template photographs for remote fitting procedures of 3D printed partial hand prosthesis $(\mathrm{A}, \mathrm{B}$, and $\mathrm{C})$ and arm prosthesis (D). Dashed red lines indicate measurements boundaries over wrist and elbow joints. A: Wrist extension (A1: non-affected, A2 affected), B: Wrist flexion (B1: non-affected, B2: affected), and C: Top view of hands and forearms (C1: Non-affected hand length, C2: Non-affected hand width, C3: Non-affected forearm length, C4: Non-affected forearm width, C5: Affected partial hand length, C6: Affected partial hand width, C7: Affected forearm length, C8: Affected forearm width). D: Top view of the entire arms (D1: Non-affected hand length, D2: Non-affected hand width, D3: Non-affected forearm length, D4: Non-affected forearm width, D5: Non-affected upper arm length. D6: Non-affected upper arm width, D7: Affected forearm length, D8: Affected forearm width, D9: Affected upper arm length, D10: Affected upper arm width).

Figure 1. Illustration of a photograph imported as a plane and overlaid under the palm section scaled at $140 \%$ for a 16 year old research participant (A). Overlay of a 3D printed arm prostheses model scaled at $99 \%$ for a 7 -year old child with a trans-radial reduction in the left arm (B).

Figure 3. The 3D printed partial hand prosthesis (Cyborg Beast 2). A. The hand prosthesis in the open position. Elastic cords placed inside the dorsal aspect of the fingers provide passive finger extension. B. Finger flexion was driven by non-elastic cords along the palmar surface of each finger and was activated through $20^{\circ}$ wrist flexion of the residual functional joint. The red arrow shows the direction of wrist flexion to close the fingers and produce a functional grasp.

Figure 4. The 3D Printed arm prostheses. A. The trans-radial arm prosthesis in the open position. Elastic cords placed inside the dorsal aspect of the fingers provide passive finger extension. B. Finger flexion was activated through $10-20^{\circ}$ of elbow flexion of the residual functional joint. The red arrow shows the direction of elbow flexion to close the fingers and produce a functional grasp. The wrist can be manually adjusted. 